\title{
Action as Entropy
}

\author{
John L. Haller Jr.
}

Chief Data Scientist, CCC Information Services, Chicago, IL, USA, E-Mail: jlhaller@alumni.princeton.edu; Tel.: +1-650-776-3001

Published: 12 November 2015

\begin{abstract}
I explore the entropy of a diffusing particle. Further examination of the mutual information of the particle and the vacuum, yields a derivation of the "action" of the particle being equal to the entropy. I argue the second law of thermodynamics is the justification for the principle of least action.
\end{abstract}

Keywords: Entropy; action; quantum of action; discrete space; discrete time; diffusion

\section{Introduction}

Information theory is closely related to physics through both quantum mechanics and thermodynamics. By looking at the quantum diffusion of a massive particle and calculating its entropy conditioned on its interaction with the vacuum, it is possible to derive the "action" of the particle.

The "action" of the particle is as foundational to physics and its history as are the concepts of thermodynamics [1]. By showing the unification of entropy and action we see what could be the tip of the iceberg.

\section{Diffusion}

As a simple model for the diffusion of a particle, I suggest that the minimum uncertainty wave packet undergoes the Bernoulli process. With this model we calculate the entropy. 


\subsection{Bernoulli Process}

Introducing the Bernoulli process as reviewed by Chandrasakhar and Reif [2,3], we can look at the average displacement where $\beta$ is the probability of stepping to the right, $N$ is the number of steps and $\delta x$ is the step size, $\overline{x(N)}$ is the average displacement and $c t$ is the speed of light times time. We have,

$$
\overline{x(N)}=(2 \beta-1) \delta x N=(2 \beta-1) c t
$$

Or ${ }^{1}$

$$
(2 \beta-1)=v / c
$$

When you correctly account for the variance from diffusion in both the spatial $\left(\Delta x(N)_{\beta}\right)^{2}$ and momentum domain, $\Delta p(N)_{\beta}$, you get, $\left(\Delta x_{B}(N)_{\beta}\right)^{2}$. We see the variance of a particles location as function of number of steps $[2,4]$,

$$
\begin{aligned}
\left(\Delta x_{B}(N)_{\beta}\right)^{2} & =\left(\Delta x(N)_{\beta}\right)^{2}+\left(\frac{\Delta p(N)_{\beta}}{m} \delta t N\right)^{2} \\
& =4 \beta(1-\beta)\left(2(\delta x)^{2} N\right)
\end{aligned}
$$

Plugging in the equation above between $v$ and $\beta$

$$
\left(\Delta x_{B}(N)_{v}\right)^{2}=2\left(1-\left(\frac{v}{c}\right)^{2}\right)(\delta x)^{2} N
$$

\subsection{Minimum Uncertainty Wave Packet}

Consider a minimum uncertainty wave packet, or Gaussian wave packet superimposed onto a discrete (left / right) step. When one considers the Hirshmann entropy of the Gaussian and adds it to the binary entropy of the Bermoulli process the result is one natural unit of entropy per step when $\beta=1 / 2$. Let's see how this plays out.

Hirshman [5] proposed that to properly measure the entropy contained in a pair of distributions linked through the Fourier Transform (FT) one must add the differential entropy of the probability distribution in the time domain to the differential entropy of the probability distribution in the standard frequency domain. Hirshman found that any FT pair contained at least $\log (e / 2)$ of information and that the Gaussian has exactly $\log (e / 2)$.

I suggest that addition entropy from each step of the Bernoulli process can be added to the wave function. If we break apart the Gaussian into a positive and negative state (which models the Bernoulli process) we can see this more formally.

The positive eigenvalue state is

$$
\phi_{+}(x)=\frac{1}{\sqrt[1 / 4]{2 \pi(\Delta x)^{2}}} e^{\frac{-(x-\delta x)^{2}}{4(\Delta x)^{2}}}
$$

And for the negative eigenvalue,

\footnotetext{
${ }^{1}$ Notice that this equation is mathematically nice since $\beta \in[0,1]$ and $v \in[-c, c]$
} 


$$
\phi_{-}(x)=\frac{1}{\sqrt[1 / 4]{2 \pi(\Delta x)^{2}}} e^{\frac{-(x+\delta x)^{2}}{4(\Delta x)^{2}}}
$$

If $\Delta x \ll \delta x$ the two functions don't overlap, they don't interfere and thus according to Feynman [1] it's their probability distributions that add not the probability amplitudes (or wave functions). See Figure 1. If the probability the particle steps to the right is $\beta$, the resulting probability distribution for the spatial domain $p_{\phi}(x)$ is,

$$
p_{\phi}(x)=\beta\left|\phi_{+}(x)\right|^{2}+(1-\beta)\left|\phi_{-}(x)\right|^{2}
$$

Taking the Fourier Transform, where $k$ is the wave number, we have

$$
\begin{aligned}
& \Phi_{+}\left(\frac{k}{2 \pi}\right)=\int \phi_{+}(x) e^{-i k x} d x \\
& \Phi_{-}\left(\frac{k}{2 \pi}\right)=\int \phi_{-}(x) e^{-i k x} d x
\end{aligned}
$$

The resulting probability distribution (with the integrand for clarity) for the standard wave vector domain, $P_{\Phi}(k / 2 \pi)$ is,

$$
P_{\Phi}\left(\frac{k}{2 \pi}\right) d\left(\frac{k}{2 \pi}\right)=\beta\left|\Phi_{+}\left(\frac{k}{2 \pi}\right)\right|^{2} d\left(\frac{k}{2 \pi}\right)+(1-\beta)\left|\Phi_{-}\left(\frac{k}{2 \pi}\right)\right|^{2} d\left(\frac{k}{2 \pi}\right)
$$

Given the modulation properties of the FT, $\left|\Phi_{+}\right|^{2}=\left|\Phi_{-}\right|^{2}$ and with $2 \Delta k \Delta x=1, P_{\Phi}(k / 2 \pi)$ reduces to

$$
P_{\Phi}\left(\frac{k}{2 \pi}\right) d\left(\frac{k}{2 \pi}\right)=\sqrt{\frac{2 \pi}{(\Delta k)^{2}}} e^{\frac{-k^{2}}{2(\Delta k)^{2}}} d\left(\frac{k}{2 \pi}\right)
$$

\subsection{Hirshman Sum}

Now using Hirshman's sum the resulting entropy, $H_{c}$ is the sum of the differential entropy, $h()$, in the time domain plus the differential entropy in the standard frequency domain.

$$
\begin{gathered}
H_{c}=h\left(p_{\phi}(x)\right)+h\left(P_{\Phi}\left(\frac{k}{2 \pi}\right)\right) \\
=H_{2}(\beta)+\frac{1}{2} \log \left(2 \pi e(\Delta x)^{2}\right)+\frac{1}{2} \log \left(e(\Delta k)^{2} / 2 \pi\right)=H_{2}(\beta)+\log (e / 2)
\end{gathered}
$$

Where the binary entropy is, $H_{2}(\beta)$

$$
H_{2}(\beta)=-\beta \log (\beta)-(1-\beta) \log (1-\beta)
$$



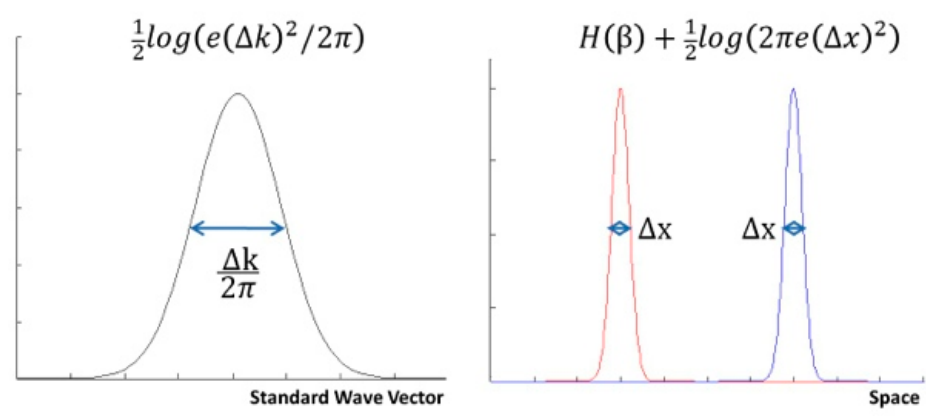

Figure 1. Probability for Gaussian with non-overlapping positive and negative states.

Notice that when $\beta=1 / 2, H_{c}=1$ natural unit of entropy per step.

\section{Joint Entropy: Particle, Vacuum}

Now let us consider separating the conditional entropy of the particle given the vacuum and the mutual information with the vacuum.

\subsection{Conditional entropy of a kinetic particle}

I claim that the entropy calculated in section $2.3, H_{c}$, is actually the entropy of the particle conditioned on the vacuum.

$$
H_{c}=H(\text { particle } \mid \text { vacuum potential })
$$

This is because the particle has interacted with the vacuum potential to make it kinetic and given it a velocity. If the particle has no more interaction with a force or the vacuum, the particle will continue to move with the same velocity as dictated by Newton's first law [1]. The resulting biased diffusion is thus a function of the state of the particle after its interaction with the vacuum.

If we return to our result from section 2.3 and with $(2 \beta-1)=v / c \ll 1$, we can Taylor expand the logarithm in $H_{2}(\beta)$ and reduce it to $\log (2)-(v / c)^{2} / 2$ for a total entropy of the particle of $1-$ $(v / c)^{2} / 2$ in natural units per step. With the step occurring each quantum unit of time, $\delta t=\hbar / 2 m c^{2}[4]$, the conditional entropy, $H_{C}$, rate is

$$
\frac{\mathrm{d} H_{C}}{\mathrm{~d} t}=\frac{2}{\hbar}\left(m c^{2}-\frac{m v^{2}}{2}\right)=\frac{2}{\hbar}\left(m c^{2}-K\right)
$$

\subsection{Mutual information: particle and vacuum}

Let's now consider the mutual information between the vacuum and the particle. Using the Gaussian channel [6] where the signal is a step in the vacuum Bernoulli process and the noise is the diffusion of the particle over the relaxation time, $\tau=\hbar / 2 k_{B} T$ [7-9], the incremental information is $\mathrm{d} C$

$$
\mathrm{d} C=\frac{1}{2} \log \left(1+\frac{S}{N_{D}}\right)
$$

The signal, $S$, is $\left(\Delta x_{B}(N)_{v}\right)^{2}$ from section 2.1 with $N=1$

$$
S=\left(\Delta x_{B}\right)_{\text {Vaccum }}^{2}=2\left(1-\left(\frac{v}{c}\right)^{2}\right)\left(\delta x_{\text {vacuum }}\right)^{2}
$$


Where $\delta x_{\text {vacuum }}$ is the spatial step of the vacuum.

We can calculate the noise, $N_{D}$, by using Einstein's kinetic relation for the diffusion constant, $D_{\mu}$ [79], with the diffusion over the relaxation time, $\tau$,

$$
N_{D}=2 D_{\mu} \tau=2 \mu k_{B} T \tau=\frac{v \hbar}{F}
$$

We now make the interpretation that a step in the vacuum Bernoulli process, $\delta x_{\text {vacuum }}$, divided by the speed of light, is the time differential $\mathrm{d} t$. Since $S \ll N_{D}$ we can Taylor expand the logarithm. We should also account for both the positive and negative energy states which are the solution to Dirac's equation [10], we get a factor of 2 when calculating the differential mutual information, $I_{M}$,

$$
\mathrm{d} I_{M}=2 \mathrm{~d} C=\frac{2\left(1-\left(\frac{v}{c}\right)^{2}\right) F c^{2}(d t)^{2}}{v \hbar}
$$

With $v=\mathrm{d} x / \mathrm{d} t$, we have,

$$
\frac{\mathrm{d} I_{M}}{\mathrm{~d} t}=\frac{2 F c^{2}}{\hbar \mathrm{d} x /(\mathrm{d} t)^{2}}-\frac{2 F v \mathrm{~d} t}{\hbar}
$$

Replacing the force, $F$, as $m \mathrm{~d} x /(\mathrm{d} t)^{2}$ and $v \mathrm{~d} t=\mathrm{d} x$; we next consider conservative forces so we can replace the incremental work, $F \mathrm{~d} x$, with the potential energy, $V$, minus the reference potential, $V_{0}$.

$$
\frac{\mathrm{d} I_{M}}{\mathrm{~d} t}=\frac{2}{\hbar}\left(m c^{2}+V-V_{0}\right)
$$

To solve for the reference potential energy, $V_{0}$, I postulate that the particle and the vacuum are independent when the potential is zero, $V=0$. When the two are independent the mutual information rate is zero [6],

$$
\frac{2}{\hbar}\left(m c^{2}-V_{0}\right)=0
$$

And thus

$$
\frac{\mathrm{d} I_{M}}{\mathrm{~d} t}=\frac{2}{\hbar} V
$$

\subsection{Entropy and "Action"}

From information theory, one can prove [6] that the total self-information, $H$, is equal to the conditional entropy, $H_{c}$, plus the mutual information, $I_{M}$. Thus we have,

$$
H=H_{C}+I_{M}
$$

Or,

$$
H=\frac{2}{\hbar} \int\left(m c^{2}-(K-V)\right) d t=\frac{2}{\hbar} \int\left(m c^{2}-\mathcal{L}\right) d t
$$

We now see that the self-information of the particle given is equal to the time integral of the mass energy minus the Lagrangian, $\mathcal{L}$, with a proportionality being the quantum of "action". 


\subsection{Principle of least action}

For $v \ll c$ (which is an assumption we used to Taylor expand the logarithm), $m c^{2}$ will be a constant and thus the integrand $\left(m c^{2}-\mathcal{L}\right)$ is an equivalent Lagrangian to $\mathcal{L}$, except with the minus sign [1].

I will not attempt to review the calculus of variations or Hamilton's principle [1,11] here (which allows one to derive the equations of motion); however I will state that the principle of least action is now seen as a principle of maximum entropy. One could go further and suggest that the principle of least action is a consequence of the second law of thermodynamics driving self-information or entropy to a maximum $[1,3]$.

\section{Conclusion}

In this letter, I have suggested that the conditional entropy rate of diffusion is proportional to the mass of the particle minus the kinetic energy. Further, the mutual information between the particle and the vacuum is equal to the potential energy. This leads us to the conclusion that the entropy of the particle (in natural units) is equal to the energy times time minus the "action" of the particle (in units of the quantum of action).

In an attempt to keep this letter brief, I will not go into all the implications I see, but rather mention two and provide the reader a few references [4,12-14] for more.

First, if we unify "action" and entropy, then entropy should be quantized like "action" is. The entropy of a particle, the entropy of a degree of freedom should be quantized to integer natural units.

Second, if "action" is finite, then entropy should be finite, which means precision to a continuous value is not possible. This implies that space-time from a discrete set of possible values.

I lastly argue that further investigation has the potential to unify two basic tenants of physics and has the potential bring insight to previously unseen solutions.

\section{Acknowledgements}

JLH is grateful to his wife and two boys and would like to thank all of those who have helped him shape this work over the last 20 years.

\section{Conflicts of Interest}

"The authors declare no conflict of interest".

\section{References}

1. R. Feynman, Lectures on Physics, Addison-Wesley Publishing, Reading, 1965

2. Chandrasekhar, Reviews of Modern Physics, Vol. 15, 1943., pp. 1-89

3. Reif, Fundamentals of Statistical and Thermal Physics, McGraw Hill, Boston, MA 1965

4. J. Haller Jr., "Measuring a Quantum System's Classical Information," Journal of Modern Physics, Vol. 5 No. 1, 2014, pp. 8-16. doi: 10.4236/jmp.2014.51002

5. Hirshman, I. I., “A Note on Entropy,” American J. of Mathematics, January 1957, 79, No1, p. 152 
6. T. Cover \& J. Thomas, Elements of Information Theory, John Wiley \& Sons Inc., New York, NY, 1991

7. R Kubo, "The fluctuation-dissipation theorem," Rep. Prog. Phys. 1966, 29255

8. J. Haller Jr., "Entropy Rate of Thermal Diffusion," Journal of Modern Physics, Vol. 4 No. 10, 2013, p. 1393-1399 doi:10.4236/jmp.2013.410167

9. E. Nelson, "Derivation of the Schrödinger Equation from Newtonian Mechanics," Phys. Rev., 1966, Vol. 150 No. 4 pp. 1079

10. P. A. M. Dirac, The Principles of Quantum Mechanics, 4th Edition, Oxford University Press, Oxford, 1958

11. Apostol, Calculus, Volume II $2^{\text {nd }}$ Ed., John Wiley \& Sons New York, NY, 1969

12. J. Haller Jr., "Information Mechanics", arXiv:physics/0106081

13. J. Haller Jr., "Dark Particles Answer Dark Energy," Journal of Modern Physics, Vol. 4 No. 7A1, 2013, p. 85-95 doi: 10.4236/jmp.2013.47A1010

14. J. Haller Jr., "A Stochastic Explanation of Newton's Law and Coulombs' Law," GJSFR Volume 14 Issue 2 Version 1.0

(C) 2015 by the authors; licensee MDPI, Basel, Switzerland. This article is an open access article distributed under the terms and conditions of the Creative Commons Attribution license (http://creativecommons.org/licenses/by/3.0/). 